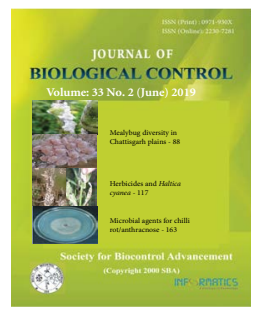

\title{
Biodiversity of mealybugs, their host range and bio-control agents associated in different districts of Chhattisgarh plains
}

\author{
CHANDRAMANI SAHU ${ }^{*}$, SUNIL JOSHI ${ }^{2}$ and JAYA LAXMI GANGULI ${ }^{1}$ \\ ${ }^{1}$ Department of Entomology, College of Agriculture, Indira Gandhi Agricultural University, Raipur - 492012, Chhattisgarh, \\ India \\ ${ }^{2}$ ICAR-National Bureau of Agricultural Insect Resources, Bengaluru - 560024, Karnataka, India \\ *Corresponding author E-mail: cmento2111@gmail.com
}

ABSTRACT: The survey of host range of mealybugs in five districts of Chhattisgarh plain regions namely Rajnandgaon, Balod, Dhamtari, Mahasamund and Raipur districts in which 132 plant species were observed. Among these, 72 plant species belonging to 28 families were identified as hosts out of which two species were of pulses (2.78\%), one oilseed (1.39\%), ten vegetables (13.89\%), twelve fruits (16.67\%), one fibre $(1.39 \%)$, one sugarcane crop (1.39\%), nineteen ornamentals (26.39\%), fifteen medicinal (20.83\%), two forest trees $(2.78 \%)$ and nine plants under weeds (12.50\%). The highest number of host plants of mealybugs were recorded in the family Malvaceae and Asteraceae followed by Fabaceae, Apocynaceae, Solanaceae, Rosaceae, Amaranthaceae and Lamiaceae. Among 72 plant species, 36 species of host plants were found having low infestation, 12 species had medium infestation and 24 plant species were observed to be highly infested by mealybugs. A total of eight mealybug species namely Ferrisia virgata Cockerell, Maconellicoccus hirsutus Green, Nipaecoccus viridis Newstead, Paracoccus marginatus Williams and Granara de Willink, Phenacoccus solenopsis Tinsley, Rastrococcus iceryoides Green and seven species of their natural enemies i.e., Cheilomenes sexmaculata, Brumoides suturalis, Scymnus sp., Chrysoperla sp., spiders as a general predator and Aenasius sp. and Pseudleptomastix mexicana as parasitoid were observed during the present studies. Among the Mealybugs species, $P$. solenopsis Tinsley was noticed as the most dominant species which observed on 14 species of different host plants infested with maximum 46.66 percent.

KEY WORDS: Host plants, mealybugs, natural enemies

(Article chronicle: Received: 16-12-2018; Revised: 20-02-2019; Accepted: 15-03-2019)

\section{INTRODUCTION}

Mealybug is the common name of insects belonging to Pseudococcidae, a family constituting of unarmored scale insects found in moist, warm climates. They are considered pests as they feed on plant juices of greenhouse plants, house hold garden plants and subtropical trees (Jahn et al., 2003). In recent years, mealy bug, Maconellicoccus hirsutus (Green) has become a serious menace to successful cultivation of different fruit crops in India. The mealybug species are widespread throughout the world. It has been found on a relatively wide variety of host plants like mango, grapevines, citrus, custard apple, sapota, cashew, pineapple and ornamentals like hibiscus, croton, ferns, cacti, gardenias and orchids etc. Besides these, mealybug infestation has also been reported in storage of tuber crops like aerial yam, elephant foot yam, colocasia, cassava etc. (Keraba, 2011). Feeding due to mealybugs, reduces plant vigour and the honeydew secreted promotes the growth of a black sooty mould that interferes with photosynthesis and affects fruit quality
(Gullan and Martin, 2009). Biological control of mealybugs has been widely studied since the early twentieth century, due to the economic importance and invasive habits of this family (McKenzie, 1967). They have a wide variety of predators, including: Coccinellids, Coleopterans, lacewings of the families Chrysopidae, Coniopterygidae and Hemerobiidae, flies of the families Cecidomyiidae and Chamaemyiidae, Anthocorids bugs, Lycaenids, lepidopterans, and Phytoseiid mites (Franco et al., 2000).

In Chhattisgarh, mealybug infestation was observed on almost all crops including cereals, legumes, oilseeds, vegetables, fruits, ornamentals, medicinal and weeds. So far, no proper documentation and identification of the various species of mealybugsattacking on different plants and their related natural enemies is available, looking to the above facts the present studies were taken up, to record, identify and search for associated bio-agents for eco-friendly management of the pest. 


\section{MATERIALS AND METHODS}

The present investigation was conducted in Chhattisgarh plain regions and the Biological control laboratory of the Department of Entomology, College of Agriculture, Indira Gandhi Krishi Vishwavidyalaya, Raipur, (Chhattisgarh) during the 2015-16 and 2016-17. Survey of different host range of mealybugs attacking on commonly grown cereals, pulses, oilseeds, vegetables, flower, fruits, ornamental and forest trees conducted at five districts of Chhattisgarh plain regions viz. Raipur, Rajnandgaon, Balod, Dhamtari and Mahasamund. From each district, four villages were selected for observation of the various host plants of mealybugs. Nature of damage, location of occurrence and plant part infected with mealybugs were also recorded. The level of damage was calculated by placing all the host plants into three categories viz., category I - low ( $1-15 \%$ plant area damaged), category II - moderate (16 - 50\% plant area damaged) and category III - high ( $>50 \%$ plant area damaged) (Selvaraju and Sakthivel, 2011). For identification of mealybugs, insects were collected from different parts of the plants like leaves, stems, flower and seeds. The samples consisted mostly of adult females and immature instars of mealybug. The collected samples were preserved in $70 \%$ ethanol for transporting to the laboratory. In case of natural enemies i.e., parasites, parasitoids and predators, plant parts with infested mealybugs were kept separately in cages at room temperature to facilitate emergence of natural enemies. Emerged natural enemies were also preserved in $70 \%$ ethanol solution. All the collected specimens were sent to National Bureau of Agricultural Insect Resources, Bengaluru for identification.

\section{RESULTS AND DISCUSSION}

\section{Survey of host plants of mealybugs}

The field survey of host plants of mealybugs was carried out from July, 2015 to June, 2017 in five districts of Chhattisgarh plain regions namely Rajnandgaon, Balod, Dhamtari, Mahasamund and Raipur district in which 132 plant species were observed for the survey of host range of mealybugs. A total of 72 plant species belonging to 28 plant families were identified as hosts (Table 1).

The mealybugs have a wide host range and great potential to cause damage to economically important agricultural, horticultural, ornamental and forest plants. The host range of mealybugs was sampled in twenty villages from five districts in plain region. The observed plant species were categorized into cereals, pulses, oilseed, vegetable, fruit, fibre, sugarcane, ornamental, medicinal, forest trees and weeds on the basis of economical value. In the comparative studies of host range of mealybugs in five different districts of Chhattisgarh plains revealed that plant species such as aonla, congress grass, guava, hibiscus, mango, okra and papaya were identified as common hosts of mealybugs. They were observed in all five selected districts whereas some plant hosts were found in particular area such as cashew, chotadatura, lantana, water hyacinth observed to be infested with mealybugs in only Raipur while cow pea, jaribhaji and sunflower were recorded as host plants in Rajnandgaon district. Overall, from the present studies a total of 72 plant species belonging to 28 families were recorded as hosts for mealybugs. Among these, two species were of pulses comprising $2.78 \%$, one oilseed $(1.39 \%)$, ten vegetables $(13.89 \%)$, twelve fruits $(16.67 \%)$, one fibre $(1.39 \%)$, one sugarcane crop $(1.39 \%)$, nineteen ornamentals $(26.39 \%)$, fifteen medicinal (20.83\%), two forest trees $(2.78 \%)$ and nine plants under weeds $(12.50 \%)$ (Table 2). The highest number of host plants of mealybugswere recorded in the family Malvaceae (8 plant species) and Asteraceae ( 8 plant species) followed by Fabaceae (7 plant species), Apocynaceae (7 plant species), Solanaceae (5 plant species), Rosaceae (4 plant species), Amaranthaceae (3 plant species) and Lamiaceae (3 plant species). The rest of the families had just two or less than two plant species as representatives (Table 3). On the basis of level of infestation of host plant species, 36 species were found having low infestation (e.g., pigeon pea, bottle gourd, chechbhaji, tamarind, oak etc.), 12 species had medium infestation (e.g., khattabhaji, strawberry, marigold, curry leaf, akarkara etc.) and 24 plant species (e.g., tomato, brinjal, papaya, aonla, hibiscus, croton etc.) were observed to be highly infested by mealybugs (Table 4).

\section{Species of mealybugs and their natural enemies}

The samples of mealybugs collected from five districts of Chhattisgarh plains namely Rajnandgaon, Balod, Dhamtari, Mahasamund and Raipur during 2015-16 to 2016-17 under the survey program consisted mostly of adult females and immature instars along with various species of natural enemies i.e., parasites, parasitoids and predators. The collected specimens were sent to National Bureau of Agricultural Insect Resources (NBAIR), Bengaluru for identification. During the present investigationsa total of eight mealybugs species namely Ferrisia virgata Cockerell, Maconellicoccus hirsutus Green, Nipaecoccus viridis Newstead, Paracoccus marginatus Williams and Granara de Willink, Phenacoccus solenopsis Tinsley, Rastrococcus iceryoides Green, Coccidohystrix iceryoides Green, Saccharicoccus sacchari Cockerell (Table 5 and Fig. 1) and seven species of their natural enemies i.e., Cheilomenes sexmaculata, Brumoides suturalis Scymnus sp., Chrysoperla sp., spiders, Aenasius sp. and Pseudleptomastix mexicana were recorded (Table $7 \&$ Fig. 2). Six species of mealybugs viz. F. virgata, M. hirsutus, 
Table 1. Host plants of mealybugs recorded in plain regions of Chhattisgarh during 2015-16 and 2016-17 on the basis of plant category and infestation level

\begin{tabular}{|c|c|c|c|c|c|}
\hline S1.No. & Common name & Botanical name & Family & Plant category & Infestation level \\
\hline 01. & Soybean & G. $\max$ & Fabaceae & Pulse & M \\
\hline 02. & Pigeon pea & C. cajan & Fabaceae & 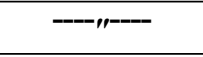 & $\mathrm{L}$ \\
\hline 03. & Sunflower & H. annuus & Asteraceae & Oilseeed & $\mathrm{L}$ \\
\hline 04. & Tomato & S. lycopersicum & Solanaceae & Vegetable & $\mathrm{H}$ \\
\hline 05. & Brinjal & S. melongena & Solanaceae & -"י- & $\mathrm{H}$ \\
\hline 06. & Cow pea & V. unguiculata & Fabaceae & 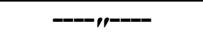 & $\mathrm{L}$ \\
\hline 07. & Potato & S.tuberosum & Solanaceae & --י-י & $\mathrm{H}$ \\
\hline 08. & Okra & A.esculentus & Malvaceae & -"ו- & $\mathrm{H}$ \\
\hline 09. & Bottle gourd & L. siceraria & Cucurbitaceae & -ים & $\mathrm{L}$ \\
\hline 10. & Khattabhaji & H. cannabinus & Malvaceae & -"י- & M \\
\hline 11. & Jaribhaji & A. dubius & Amaranthaceae & --"- & $\mathrm{M}$ \\
\hline 12. & Chechbhaji & C. actutangulas & Malvaceae & -" & $\mathrm{L}$ \\
\hline 13. & Kanda bhaji & Ipomia sp. & Convolvulaceae & --"י- & $\mathrm{L}$ \\
\hline 14. & Cashew & A. occidentale & Anacardiaceae & Fruit & M \\
\hline 15. & Apple & M. domestica & Rosaceae & -"- & $L$ \\
\hline 16. & Strawberry & F. ananassa & Rosaceae & ---"-- & M \\
\hline 17. & Guava & P. guajava & Myrtaceae & --"- & $\mathrm{H}$ \\
\hline 18. & Ber & Z. mauritiana & Rhamnaceae & -"- & $\mathrm{L}$ \\
\hline 19. & Papaya & C. papaya & Caricaceae & --"- & $\mathrm{H}$ \\
\hline 20. & Mango & M. indica & Anacardiaceae & --ール-- & $\mathrm{L}$ \\
\hline 21. & Tamarind & T. indica & Fabaceae & -"- & $\mathrm{L}$ \\
\hline 22. & Aonla & E. officinalis & Euphorbiaceae & --"-- & $\mathrm{H}$ \\
\hline 23. & Citrus & C. vlemon & Rutaceae & -יו- & $\mathrm{L}$ \\
\hline 24. & Custard apple & A. squamosa & Annonaceae & --" & $\mathrm{H}$ \\
\hline 25. & Pomegranate & P. granatum & Punicaceae & --"-- & $\mathrm{L}$ \\
\hline 26. & Cotton & Gossypium sp. & Malvaceae & Fibre & $\mathrm{H}$ \\
\hline 27. & Sugarcane & S. officinarum & Poaceae & Sugar & $\mathrm{L}$ \\
\hline 28. & Temple tree & P. alba & Apocynaceae & Ornamental & $\mathrm{L}$ \\
\hline 29. & Hibiscus & Hibiscus sp. & Malvaceae & --"- & $\mathrm{H}$ \\
\hline 30. & Marigold & T. erecta & Asteraceae & --"»--- & $\mathrm{M}$ \\
\hline 31. & Kaner & Nerium sp. & Apocynaceae & --"-- & $\mathrm{L}$ \\
\hline 32. & Satpatia & A. scholaris & Apocynaceae & -י-י & $\mathrm{L}$ \\
\hline 33. & Croton & C. variegatum & Euphorbiaceae & --"»--- & $\mathrm{H}$ \\
\hline 34. & Periwinkle & C. roseus & Apocynaceae & --"-י & $\mathrm{L}$ \\
\hline 35. & Dumb cane & D. spp. & Araceae & -י-י & $\mathrm{L}$ \\
\hline 36. & Mani plant & S. podophyllum & Araceae & --"י- & $\mathrm{H}$ \\
\hline 37. & Rose & Rosa sp. & Rosaceae & --"-- & $\mathrm{L}$ \\
\hline 38. & China rose & R. chinensis & Rosaceae & --״-- & $\mathrm{H}$ \\
\hline 39. & Phlox & Phlox sp. & Polemoniaceae & --" & $\mathrm{L}$ \\
\hline 40. & Hollyhock & A. rosea & Malvaceae & --"- & $\mathrm{L}$ \\
\hline 41. & Verbena & V. officinalis & Verbenaceae & --י--י & $\mathrm{L}$ \\
\hline 42. & Candy tuff & I. sempervirens & Brassicaceae & 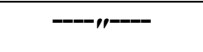 & $\mathrm{L}$ \\
\hline
\end{tabular}




\begin{tabular}{|c|c|c|c|c|c|}
\hline 43. & Goldenrod & S. virgaurea & Asteraceae & -י- & $\mathrm{L}$ \\
\hline 44. & Champa & M. champaca & Magnoliaceae & -"י- & $\mathrm{L}$ \\
\hline 45. & $\begin{array}{l}\text { Amaranthus } \\
\text { garden plants }\end{array}$ & Amaranthus sp. & Amaranthaceae & -י-י-י & $\mathrm{H}$ \\
\hline 46. & Chrysanthemum & Chrysanthemum sp. & Asteraceae & --י- & $\mathrm{L}$ \\
\hline 47. & Adusa & A. vasica & Acanthaceae & Medicinal & $\mathrm{L}$ \\
\hline 48. & Bhringraj & W. chinensis & Asteraceae & -"ח- & $\mathrm{H}$ \\
\hline 49. & Bawachi & P. corlifolia & Fabaceae & --י-י & $\mathrm{H}$ \\
\hline 50. & Tulsi & O. tenuiflorum & Lamiaceae & 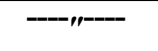 & $\mathrm{H}$ \\
\hline 51. & Samandersokh & A. nervosa & Convolvulaceae & --י- & $\mathrm{H}$ \\
\hline 52. & Ashwagandha & W. somniferous & Solanaceae & --"- & $\mathrm{H}$ \\
\hline 53. & Sarpagandha & R.serpentina & Apocynaceae & --"- & $\mathrm{L}$ \\
\hline 54. & Chui mui & M. pudica & Mimosaceae & -"-י & $\mathrm{H}$ \\
\hline 55. & Patchouli & P. cablin & Lamiaceae & --"י- & M \\
\hline 56. & Nirgundi & V.negundo & Lamiaceae & --"-- & $\mathrm{H}$ \\
\hline 57. & Kasturibhindi & A. moschatus & Malvaceae & --"י- & $\mathrm{H}$ \\
\hline 58. & Betelvine & P. betel & Piperaceae & --"י- & $\mathrm{M}$ \\
\hline 59. & Curry leaf & M.koenigii & Rutaceae & --"י- & $\mathrm{M}$ \\
\hline 60. & Gudmar & G. sylvestre & Apocynaceae & --"י- & $\mathrm{M}$ \\
\hline 61. & Akarkara & S. acmella & Asteraceae & --"- & $\mathrm{M}$ \\
\hline 62. & Chirchita & A. aspera & Amaranthaceae & Weed & $\mathrm{L}$ \\
\hline 63. & Congress grass & P. hysterophorus & Asteraceae & --"י- & $\mathrm{H}$ \\
\hline 64. & Lantana & L. camara & Verbenaceae & --"י- & $\mathrm{L}$ \\
\hline 65. & Aak/ Madar & C. gigantea & Apocynaceae & --"ו- & $\mathrm{L}$ \\
\hline 66. & Dhoob grass & C. dactylon & Poaceae & --י-י- & $\mathrm{L}$ \\
\hline 67. & Baryara & S. acuta & Malvaceae & --"״-- & $\mathrm{H}$ \\
\hline 68. & Chirpoti & P. minima & Solanaceae & --" & $\mathrm{L}$ \\
\hline 69. & Chotadhatura & X. strumarium & Asteraceae & --"-- & $\mathrm{L}$ \\
\hline 70. & Water hycinth & E. crassipes & Pontederiaceae & --״-- & $\mathrm{L}$ \\
\hline 71. & Black babool & A. nilotica & Fabaceae & Forest tree & $\mathrm{L}$ \\
\hline 72. & Karanj & P. pinnata & Fabaceae & --י- & $\mathrm{L}$ \\
\hline
\end{tabular}

(L- Low infestation level, M- Medium infestation level, H- High infestation level)

Table 2. Host plants of mealybugs recorded on the basis of different plant category

\begin{tabular}{|c|c|c|c|}
\hline S1. No. & Plant category & No. of host plants & Name of host plants \\
\hline 01. & Pulse & $02(2.78 \%)$ & Soybean, Pigeon pea \\
\hline 02. & Oilseeed & $01(1.39 \%)$ & Sunflower \\
\hline 03. & Vegetable & $10(13.89 \%)$ & $\begin{array}{c}\text { Tomato, Brinjal, Cow pea, Potato, Okra, Bottle gourd, Khattabhaji, Jaribhaji, } \\
\text { Chechbhaji, Kanda bhaji }\end{array}$ \\
\hline 04. & Fruit & $12(16.67 \%)$ & $\begin{array}{c}\text { Cashew, Apple, Strawberry, Guava, Ber, Papaya, Mango, Tamarind, Aonla, } \\
\text { Citrus, Custard apple, Pomegranate }\end{array}$ \\
\hline 05 . & Fibre & $01(1.39 \%)$ & Cotton \\
\hline 06. & Sugarcane & $01(1.39 \%)$ & Sugarcane \\
\hline
\end{tabular}




\begin{tabular}{|c|c|c|c|}
\hline 07. & Ornamentals & $19(26.39 \%)$ & $\begin{array}{c}\text { Temple tree, Hibiscus, Marigold, Kaner, Satpatia, Croton, Periwinkle, Dumb } \\
\text { cane, Mani plant, Rose, China rose, Phlox, Hollyhock, Verbena, Candy tuff, Gold- } \\
\text { enrod, Champa, Amaranthus garden plants, Chrysanthemum }\end{array}$ \\
\hline 08. & Medicinals & $15(20.83 \%)$ & $\begin{array}{c}\text { Adusa, Bhringraj, Bawachi, Tulsi, Samandersokh, Ashwagandha, Sarpagandha, } \\
\text { Chui mui, Patchouli, Nirgundi, Kasturibhindi, Betelvine, Curry leaf, Gudmar, } \\
\text { Akarkara }\end{array}$ \\
\hline 09. & Weeds & $09(12.50 \%)$ & $\begin{array}{c}\text { Chirchita, Congress grass, Lantana, Aak/ Madar, Dhoob grass, Baryara, } \\
\text { Chirpoti, Chotadhatura, Water hycinth }\end{array}$ \\
\hline 10. & Forest trees & $02(2.78 \%)$ & Karanj, Black babool \\
\hline & Total & 72 & \\
\hline
\end{tabular}

Table 3. List of families of host plants of mealybugs and associated plant species recorded in Chhattisagrh plain region

\begin{tabular}{|c|c|c|c|}
\hline Sl. No. & Family & $\begin{array}{l}\text { Number of plant } \\
\text { species }\end{array}$ & Name of plant species \\
\hline 01. & Acanthaceae & 1 & Adusa \\
\hline 02. & Amaranthaceae & 3 & Chirchita, Jaribhaji, Amaranthus garden plants \\
\hline 03. & Anacardiaceae & 2 & Cashew, Mango \\
\hline 04. & Annonaceae & 1 & Custard apple \\
\hline 05. & Apocynaceae & 7 & Aak, Gudmar, Periwinkle, Satpatia, Kaner, Temple tree, Sarpagandha \\
\hline 06. & Araceae & 2 & Dumb cane, Mani plant \\
\hline 07. & Asteraceae & 8 & $\begin{array}{c}\text { Chotadhatura, Congress grass, Akarkara, Bhringraj, Marigold, Goldenrod, } \\
\text { Sunflower, Chrysanthemum }\end{array}$ \\
\hline 08. & Brassicaceae & 1 & Candy tuff \\
\hline 09. & Caricaceae & 1 & Papaya \\
\hline 10. & Convolvulaceae & 2 & Kanda bhaji, Samandersokh \\
\hline 11. & Cucurbitaceae & 1 & Bottle gourd \\
\hline 12. & Euphorbiaceae & 2 & Croton, Aonla, \\
\hline 13. & Fabaceae & 7 & Black babool, Karanj, Bawachi, Tamarind, Cow pea, Soybean, Pigeon pea \\
\hline 14. & Lamiaceae & 3 & Tulsi, Patchouli, Nirgundi \\
\hline 15. & Magnoliaceae & 1 & Champa \\
\hline 16. & Malvaceae & 8 & $\begin{array}{l}\text { Baryara, Kasturibhindi, Okra, Khattabhaji, Chechbhaji, Cotton, Hibiscus, } \\
\text { Hollyhock }\end{array}$ \\
\hline 17. & Mimosaceae & 1 & Chui mui \\
\hline 18. & Myrtaceae & 1 & Guava \\
\hline 19. & Piperaceae & 1 & Betelvine \\
\hline 20. & Poaceae & 2 & Dhoob grass, Sugarcane \\
\hline 21. & Polemoniaceae & 1 & Phlox \\
\hline 22. & Pontederiaceae & 1 & Water hycinth \\
\hline 23. & Rhamnaceae & 1 & Ber \\
\hline 24. & Punicaceae & 1 & Pomegranate \\
\hline 25. & Rosaceae & 4 & Rose, China rose, Apple, Strawberry \\
\hline 26. & Rutaceae & 2 & Curry leaf, Citrus \\
\hline 27. & Solanaceae & 5 & Tomato, Brinjal, Potato, Chirpoti, Ashwagandha \\
\hline \multirow[t]{2}{*}{28.} & Verbenaceae & 2 & Lantana, Verbena \\
\hline & Total & 72 & \\
\hline
\end{tabular}


Table 4. Host plants of mealybugs categorized on the basis of level of Infestation

\begin{tabular}{|c|c|c|c|}
\hline Sl.No. & $\begin{array}{c}\text { Level of Infestation } \\
01 .\end{array} \begin{array}{c}\text { Category I - Low } \\
(1-15 \% \text { plant area damaged })\end{array}$ & Number of host plants & Name of host plants \\
\hline 02. & $\begin{array}{c}\text { Category II - Medium } \\
(16-50 \% \text { plant area damaged })\end{array}$ & 14 & $\begin{array}{c}\text { Pigeon pea, Sunflower, Cow pea, Bottle gourd, Chechbhaji, Kanda } \\
\text { bhaji, Apple, Ber, Tamarind, Citrus, Pomegranate, Sugarcane, } \\
\text { Temple tree, Kaner, Satpatia, Periwinkle, Dumb cane, Rose, Phlox, } \\
\text { Hollyhock, Verbena, Candy tuff, Goldenrod, Champa, } \\
\text { Chrysanthemum, Adusa, Sarpagandha, Aak/ Madar, Dhoob grass, } \\
\text { Chirpoti, Chotadhatura, Water hyacinth, Black babool, Karanj }\end{array}$ \\
\hline 03. & $\begin{array}{c}\text { Category III - High } \\
(>50 \% \text { plant area damaged })\end{array}$ & $\begin{array}{c}\text { Khattabhaji, Jaribhaji, Strawberry, Cashew, Marigold, Patchouli, } \\
\text { Betelvine, Curry leaf, Gudmar, Akarkara, Mango, Chirchita, Lan- } \\
\text { tana, Croton }\end{array}$ \\
\hline Total & 24 & $\begin{array}{c}\text { Tomato, Brinjal, Potato, Okra, China rose, Hibiscus, Cotton, } \\
\text { Custard apple, Papaya, Guava, Baryara, Congress grass, Chui mui, } \\
\text { Nirgundi, Kasturibhindi, Bhringraj, Ashwagandha, Bawachi, Tulsi, } \\
\text { Samandersokh, Amaranthus garden plants, Mani plant }\end{array}$ \\
\hline
\end{tabular}

$N$. viridis, $P$. marginatus, $P$. solenopsis, $R$. iceryoides and two species of natural enemies viz. B. suturalis, $P$. mexicana were identified by NBAIR, Bengaluru. Muthulingam (2011) identified eight different species of mealybugs and among them six were identified up to species level. Identified species were P. solenopsis, Planococcus citri, F virgata, P. marginatus, M. hirsutus and Dysmicoccus neobrevipes. Nebie et al. (2016) also identified two mealybug species in mango trees infested by Icerya aegyptiaca Douglas (Hemiptera: Monophlebidae) and F. virgata Cockerell (Hemiptera: Pseudococcidae).

Among mealybug species identified, P. solenopsis Tinsley was noticed as the most dominant species observed on 14 different host plants with 46.66 percent infestation followed by $R$. iceryoides Green in six host plants with 20 percent, $P$. marginatus Williams and Granara de Willink infesting five host plants with 16.66 percent infestation, $N$. viridis Newstead recorded on three host plants with 10 percent infestation, $F$. virgata Cockerell, M. hirsutus, $C$. iceryoides and $S$. sacchari attacked only on a single host plant with 3.33 percent infestation (Table 5). Since, species of host plants namely cotton, china rose, hibiscus and karanj were observed during the survey, it was noticed that two different species of mealybugs were attacking on a single host plant at a time such as cotton infested by $P$. solenopsis, $F$. virgata and $R$. iceryoides, china rose infested by $P$. solenopsisand $P$. marginatus, Hibiscus by $P$. solenopsisand $P$. marginatus and karanj infested by P. solenopsis by an unidentified mealybug species (Table 6). Nagrare et al. (2014) carried out twenty-six random surveys that recorded five mealybug species as minor pests of cotton in India i.e. N. viridis, F. virgata,
M. hirsutus, $R$. iceryoides and $P$. tamarindus. Among these species, $N$. viridis was found to be next most widely distributed mealybug species after P. solenopsis. Sahu (2014) reported mealybugs on aonla causing heavy damage by sucking the sap of leaves, fruits and young shoots of plants at Agro-forestry farm of IGKV, Raipur (Chattisgarh).

Among natural enemies of mealybugs, $C$. sexmaculata, B. suturalis, Scymnus sp. and Chrysoperla sp., spiders as a predators and A. bambawalei, $P$. mexicana as parasitoids were observed during the survey. The data on field survey represented that $C$. sexmaculata was associated with four species of mealybugs namely $P$. solenopsis, P. marginatus, M. hirsutus and Rastrococcus iceryoides. Similarly, B. suturalis was recorded on two species of mealybugs such as $P$. Marginatus and $P$. solenopsis. Scymnus species was observed associated with three species of mealybugs namely $N$. viridis, P. solenopsis and P. marginatus. Chrysoperla sp. was seen attacking only on a single species of unidentified mealybug of aonla, E. officinalis. Spiders were observed on two species of mealybugs i.e., $P$. solenopsis and $P$. marginatus. A. bambawalei and $P$. mexicana was found parasitization $P$. solenopsis and $P$. marginatus, respectively (Table 7). Neetan and Aggarwal (2011) recorded different natural enemies from mealy bug colonies which included four coccinellid predators, one parasitoid and one Chrysoperla species. Walton and Pringle (2014) recorded natural enemies of mealybug, Planococcusficus Signoret were predatory beetle, Nephus sp. and the parasitoids, Coccidoxenoides perminutus Girault, Anagyrus sp. and 
Table 5. List of mealybugs species with their host plants recorded from various districts of Chhattisgarh plains

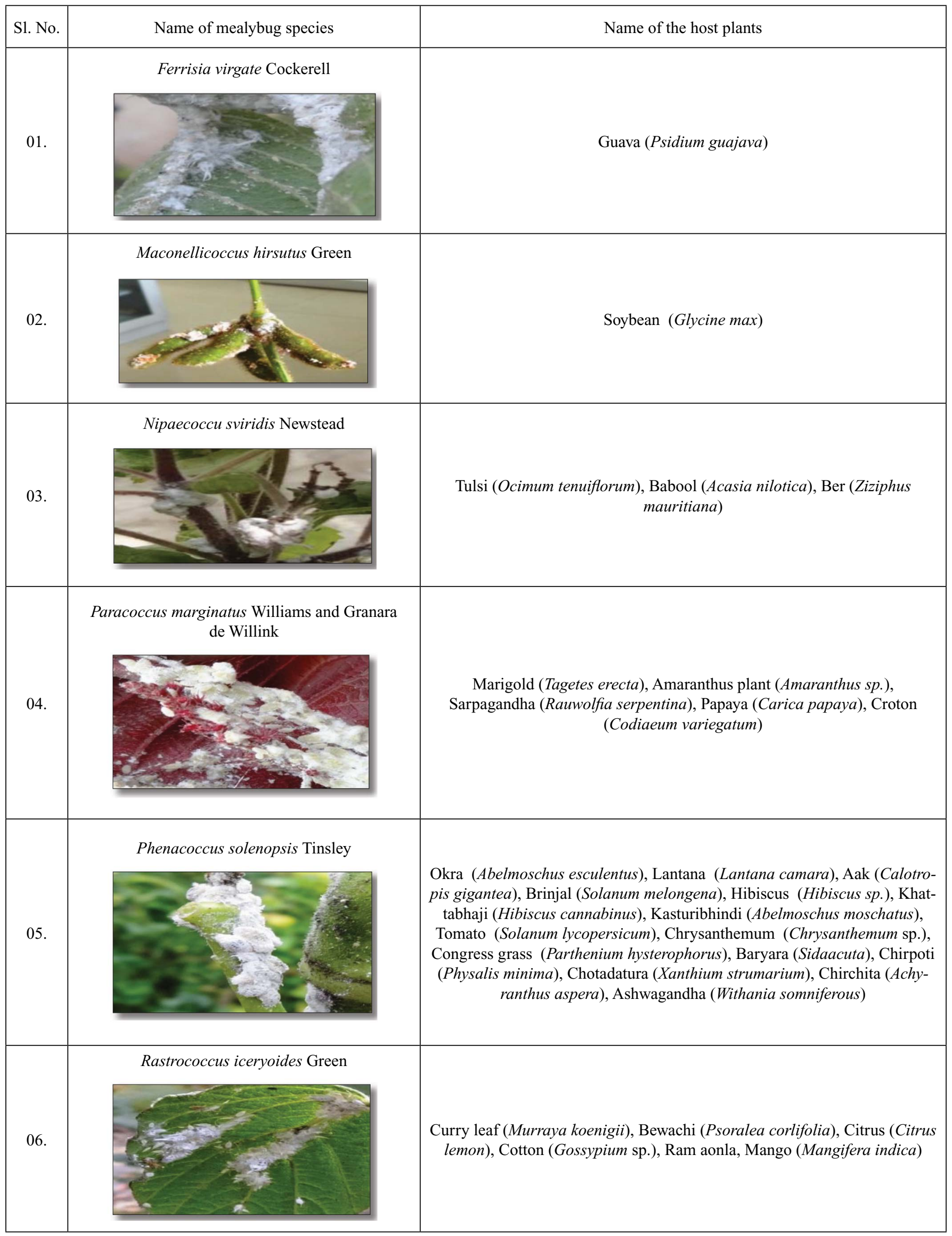




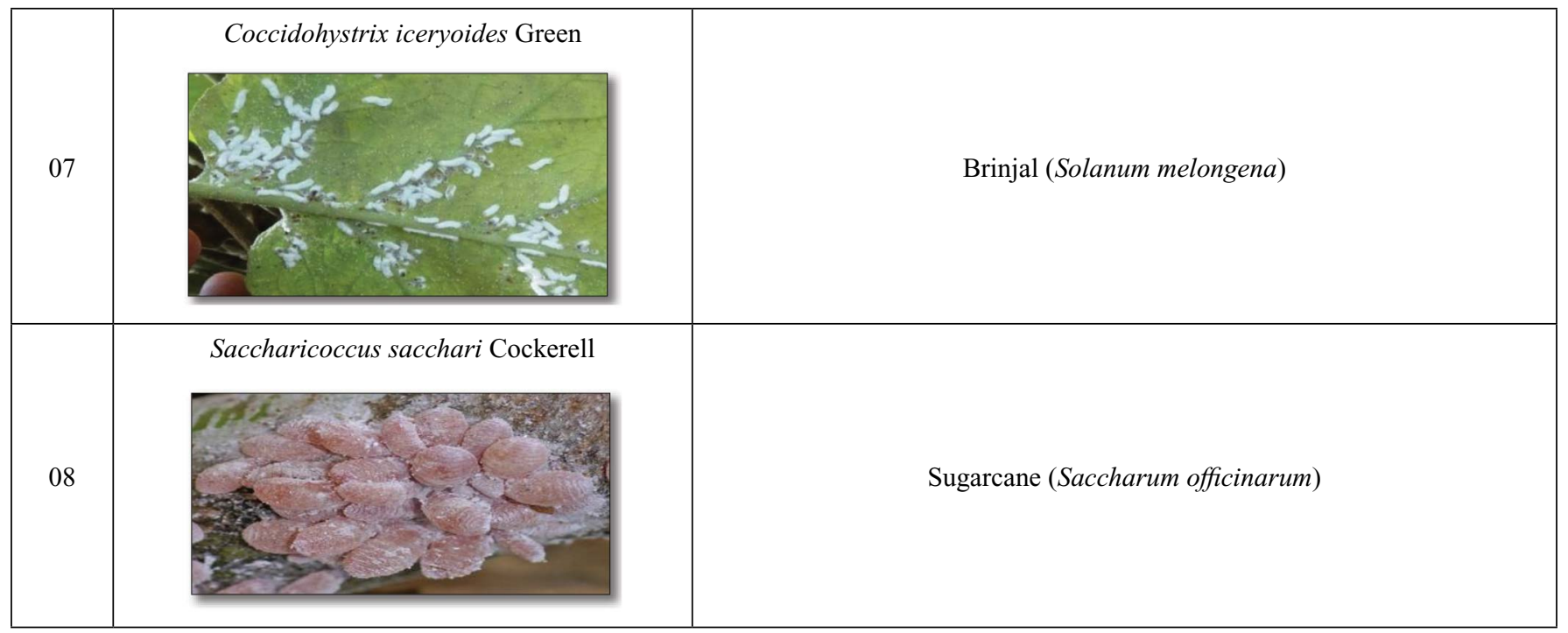

Table 6. Name of host plants of mealybugs infested by two different species observed during survey program

\begin{tabular}{|c|c|c|c|}
\hline S.N. & Name of host plants & Botanical name & Associated mealybug species \\
\hline \multirow{2}{*}{01.} & Cotton & \multirow{2}{*}{ Gossypium sp. } & Phenacoccus solenopsis \\
\cline { 3 - 4 } & China rose & Rosa chinensis & Rastrococcus iceryoides \\
\hline 02. & Hibiscus & Hibiscus sp. & Paracoccus marginatus \\
\hline 03. & Karanj & Pongamia pinnata & Paracoccus marginatus \\
\hline 04. & & & Phenacoccus solenopsis \\
\hline & & & Unidentified sp. \\
\hline
\end{tabular}

Table 7. Natural enemies on various species of mealybugs observed during 2015-16 and 2016-17

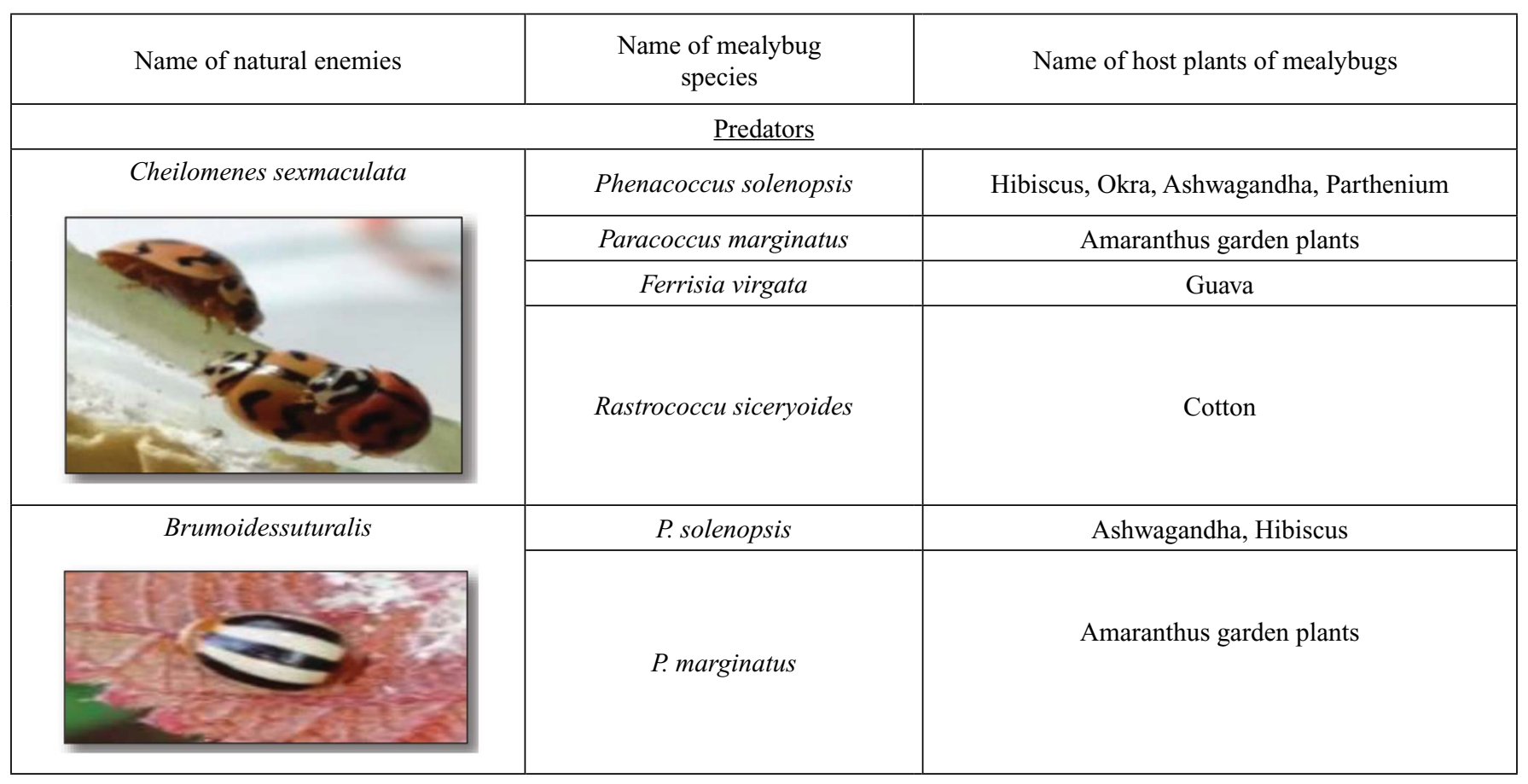




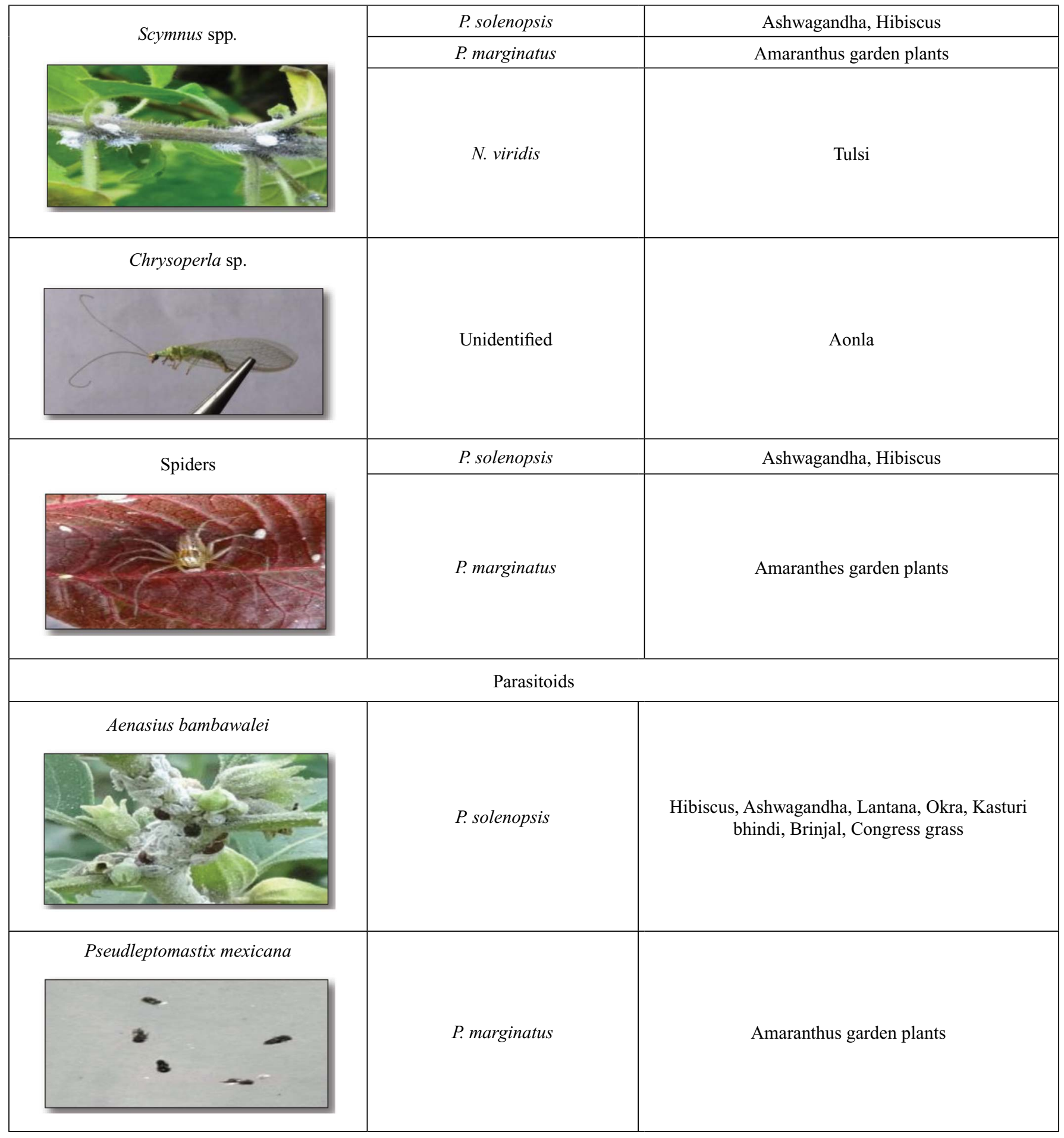

Leptomastix dactylopii Howard.

\section{ACKNOWLEDGEMENT}

The authors are thankful to Dr. V. K. Dubey, Head, Department of Entomology, and I. G. K. V. Raipur for encouragements, useful suggestion and providingnecessary facilities to conduct the experiment and special thankful to Dr.
Sunil Joshi, Principal Scientist, NBAIR, Bangalore, for the identification of mealybugs and their related natural enemies.

\section{REFERENCES}

Jahn, Gary C, Beardsley JW, and González-Hernández H. 2003. A review of the association of ants with mealybug 
wilt disease of pineapple. Proc Hawaiian Entomol Soc. 36:9-28.

Keraba SK. 2011. Mealy bugs infesting some major fruit crops of the Konkan region of Maharashtra (India). Ph.D. Thesis, Dr. Balasaheb Sawant Konkan Krishi Vidyapeeth, Dapoli. p. 13.

Gullan PJ, Martin JH. 2003. Sternorrhyncha (jumping plantlice, whiteflies, aphids and scale insects). P. 1079-1089. In: Resh VH, Cardé RT. (Eds), Encyclopedia of Insects. Amsterdam, Academic Press.

McKenzie HL. 1967. Mealybugs of California: With taxonomy, biology and control of North American species. University of California Press, Berkeley and Los Angeles.

Franco JC, Borges da Silva E, Passos de Carvalho J. 2000. Cochonilhas-algodão (Hemiptera, Pseudococcidae) associadasaoscitrinosem Portugal. ISA Press, Lisboa.

Selvaraju NG, Sakthivel N. 2011. Host plants of papaya mealybug Paracoccus marginatus Williams and Granara de Willink.) in Tamil Nadu. Karnataka J Agric Sci. 24(4): 567-569.

Nagrare VS, Kumar R, Dharajothi B. 2014. A record of five mealybug species as minor pests of cotton in India. $J$ Entomol Zool Stud. 2(4): 110-114.

Sahu C. 2014. Studies on various provenances of karanj, Pongamia pinnataL. Pierre against major insect pests intercropped with paddy under different Agroforestry cropping pattern. M.Sc. Thesis, Indira Gandhi KrishiVishwavidyala, Raipur, p.78.

Neetan, Aggarwal N. 2011. Biological control of the mealy bug, Phenacoccus solenopsis (Tinsley) on cotton. J Insect Sci. 24(4): 374-378.

Walton VM, Pringle KL. 2004. A survey of mealybugs and associated natural enemies in vineyards in the Western Cape Province, South Africa. S Afr J Enol Vitic. 25(1). https://doi.org/10.21548/25-1-2134 